\title{
DISTRIBUTED SYSTEM FOR 3D REMOTE MONITORING USING KINECT DEPTH CAMERAS
}

\author{
M. Martínez-Zarzuela ${ }^{1}$, M. Pedraza-Hueso, F.J. Díaz-Pernas, \\ D. González-Ortega, M. Antón-Rodríguez \\ ${ }^{1}$ Departmento de Teoría de la Senal y Comunicaciones e Ingenieria Telemática \\ University of Valladolid \\ marmaretel.uva.es
}

\begin{abstract}
This article describes the design and development ofa system for remote indoor $3 D$ monitoring using an undetermined number of Microsoft ${ }^{\circledR}$ Kinect sensors. In the proposed client-server system, the Kinect cameras can be connected to different computers, addressing this way the hardware limitation of one sensor per USB controller. The reason behind this limitation is the high bandwidth needed by the sensor, which becomes also an issue for the distributed system TCP/IP communications. Since traffic volume is too high, $3 D$ data has to be compressed before it can be sent over the network. The solution consists in self-coding the Kinect data into RGB images and then using a standard multimedia codec to compress color maps. Information from different sources is collected into a central client computer, where point clouds are transformed to reconstruct the scene in $3 D$. An algorithm is proposed to conveniently merge the skeletons detected locally by each Kinect, so that monitoring of people is robust to self and inter-user occlusions. Final skeletons are labeled and trajectories of every joint can be saved for event reconstruction or further analysis.
\end{abstract}

\section{KEYWORDS}

3D Monitoring, Kinect, OpenNI, PCL, CORBA, VPX, H264

\section{INTRODUCTION}

A system for remote people monitoring can be employed in a large amount of useful applications, such as those related to security and surveillance[1], human behavior analysis[2] and elderly people or patient health care[3][4]. Due to their significance, human body tracking and monitoring are study fields in computer vision that have always attracted the interest of researchers[5][6]. As a result, many technologies and methods have been proposed. Computer vision techniques are becoming increasingly sophisticated, aided by new acquisition devices and low-cost hardware data processing capabilities.

The complexity of the proposed methods can significantly depend on the way the scene is acquired. An important requirement is to achieve fine human silhouette segmentation.State-of-art technologies are really goodat this task. Apart from the techniques that use markers attached to the human body, tracking operations are carried out mainly in two ways, from 2D information or

David C. Wyld et al. (Eds) : CST, ITCS, JSE, SIP, ARIA, DMS - 2014

pp. 101-112, 2014. (C) CS \& IT-CSCP 2014

DOI : 10.5121/csit.2014.4110 
3D information [7][8]. On the one hand, 2D body tracking is presented as the classic solution; a region of interest is detected within a 2D image and processed. Because of the use of silhouettes, this method suffers from occlusions. On the other hand, advanced body tracking and pose estimation is currently being carried out by means of 3D cameras, such as binocular, Time-ofFlight (ToF) or consumer depth-cameras like Microsoft(R) Kinect[9].The introduction of lowcost depth sensors has pushed up the development of new systems based on robust segmentation and tracking of human skeletons. The number of applications built on top of depth-sensor devices is rapidly increasing. However, most of these new systems are aimed to track only one or two people thus have only direct application on videogames or human-computer interfaces.

There are some limitations to address in order to build a remote space monitoring system using consumer depth-cameras, and only a few separate efforts have been done to address these limitations. Even so, those developments do not pursue building a remote monitoring system, but covering part of the limitations in which we are also interested for our system.On the one hand, Kinect devices can capture only a quite small area, covering accurately distances only up to 3.5 meters[9]. There areproposalswhich allow to make a 3D reconstruction of spaces and objects using Kinect[10], but in themevery capturing device has to be connected to the same computer. Apart from that, these solutions cannot merge skeletonsinformation from different Kinects. The first limitation is significant, since only two or three devices can be connected to a single computer, due to the high USB bandwidth consumption of these cameras. There is another proposal that allows to send data over a network[11]. However, this application uses Microsoft SDK [9], so it only works under Windows operating system.

The 3D monitoring system presented in this paper addresses these limitations and allows using an undetermined number of Microsoft ${ }^{\circ}$ Kinect cameras, connected to an undetermined number of computers running any operating system(Windows, Linux, Mac), to monitor people in a large space remotely. The system codes the 3D information (point clouds representing the scene,human skeletons and silhouettes)acquired by each camera, so that bandwidth requirements for real-time monitoring are met.The information coming from different devices is synchronized. Point clouds are combined to reconstruct the scene in $3 \mathrm{D}$ and human skeletons and silhouettes information coming from different cameras are merged conveniently to build a system robust to self-user or inter-user occlusions. The proposed system uses low cost hardware and open source software libraries, which makes its deployment affordable for many applications under different circumstances.

Section 2 of this paper includes a general description of the tools and methods employed to develop the system, Section 3 describes de proposed system, describing important details about the 3D information coding strategy and the algorithm proposed to merge different skeletons information. Section 4 describes performance evaluation tests that were conducted in order to measure the robustness of the system. Finally, Section 5 draws the main conclusions and comments on future research tasks.

\section{TOOLS AND METHODS}

\subsection{Consumer depth-cameras}

For 3D scene acquisition, a number of devices can be used. For computer vision techniques, we can distinguish among passive and active cameras. The first include stereo devices, simulating the left and right eye in human vision:the images coming from each camera in the device are combined to generate a disparity map and reconstruct depth information[7]. In this category, some other proposals in which several passive cameras are disposed around the person or object to be reconstructed can be included. The second option consists in using an active device such as 
a TOF (Time of Flight) camera or newer consumer-depth cameras like Microsoft ${ }^{\circledR}$ Kinect or ASUS ${ }^{\circledR}$ Xtion. Despite their high depth precision, TOF cameras are expensive and provide very low resolutions. On the other side, consumer depth-cameras provide resolutions starting at 640x480 pxand $30 \mathrm{fps}$ at very affordable prices.

We would pay special interest to Kinect cameras, since they are the chosen devices for the proposed system. Microsoft $₫$ Kinect emits a structured infrared pattern of points over its field of view, which is then captured by a sensor and employed to estimate the depth of every projected point in the scene. Although Kinect was initially devised only to computer games, the interest of the computer vision communityrapidly made it possible to use the device for general purpose from a computer, even before the Microsoft ${ }^{\circledR}$ official Kinect SDK was available[9]. There is a wide variety of tools to work with Kinect. A commonly used framework for creating applications is OpenNI[12], which has been developed to be compatible with any commodity depth camera and, in combination with NiTE middleware, is able to automate tasks for user identifying, feature detection, and basic gesture recognition[13].

\subsection{Data compression}

Consumer depth cameras generate a largevolume of data. This is an importantissue, since one of the objectives of the system is the transmission of this information over a network. Therefore, data compression is necessary before sending data to a centralcomputer. There are different ways to compress data. If the data to compress is not multimedia, we can use a zip encoder, which provides lossless compression, but generates large output data and is computationally expensive. For multimedia compression, there are picture encoders like jpeg, which do not use temporal redundancy. To compress video, there are many encoders like H.264 or VP8. These encoders are able to compress data taking advantage of the temporal redundancy, thus compressed information is suitable to send over the network. However, there are not extended codecs to compress depth maps yet. One type of compression codecs used for 3D images, are those used to transmit the 3D television signal, but they are based on the compression of two images (right and left) [14], thus are not useful for our system, where 3D information is directly acquired using an active infrared device.

\subsection{CORBA}

A distributed application based on the client-server paradigm does not need to be developed using low level sockets. For the proposed system, a much more convenient approach is using a middleware such as TAO CORBA, a standard defined by OMG (Object Management Group). This middlewareallowsusinga naming service[15], that avoids the central client to know about theaddresses of each one of the servers. The aim of CORBA is to hide to the programmer thelow level complexity algorithms for data transmissionover the network. It is object-oriented and supports C++, Python, Java, XML, Visual Basic, Ada, C, COBOL, CORBA-Scripting-Language, Lisp, PL/1, Smalltalk and C\#. Besides, this middleware is chosen because it is independent of the programming language, so serverscould be programmed in Java and a client in $\mathrm{C}++$, for example. It represents a clear advantage over RMI, which can only be programmed in Java. CORBA is also cross platform, so clients and servers can be running on different operating systems.In the proposed system, the servers may be running on Windowscomputers and the client in a Linuxcomputer or in the opposite way.

\subsection{PCL: Point Cloud Library}

PCL 'Point Cloud Library'[16], is a C++ free open source computer vision library to work with $3 \mathrm{D}$ informationthat can be used in many areas such as robotics.PCL is being developed by a 
group of researchers and engineers from around the world. There are also many companies such as Toyota or Nvidiaworking to develop this powerful library [17]. The library contains algorithms for filtering, feature estimating, point cloud registration and segmentation.

Point clouds can be obtained and stored in 3D raw data files, read from 3D models or 3D cameras such as Kinect. The combination of both technologies, PCL and Kinect, is very convenient for our purpose of monitoring a space with 3D information. The library is comprised of the following modules: filters, features, keypoints, registration, kdtree, octree, segmentation, simple consensus, surface, range image, IO, visualization, common and search.

\section{PROPOSED SYSTEM}

The proposed system key feature is the fusion of 3D information coming from multiple Kinect devices, including depth information and detected skeletons. This takes place under the clientserver model, where servers are computers with attacheddevices and the client is the centralcomputer responsible for information fusion, tracking and visualization.

\subsection{General description}

Figure 1 depicts the scheme of the proposed system. A server is a computer where one or more Kinect cameras are connected. The different servers, deployed in a remote space are responsible of capturing the information coming from different regions of the scene. This information is conveniently processed and then sent to a system central computer. The large amount of information acquired by Kinect devices has to be compressed using different strategies before it can be sent over the network. The central client is in charged of reconstructing the remote space in 3D using PCL library and includes a robust algorithm for multiple detected skeletons merging. The computer interface can be used to monitor the scene in 3D in real time, label people within it and record specific users movements for further analysis[18] The system is fully scalable to any number of servers and clients, thus any number of acquiring devices and locations.

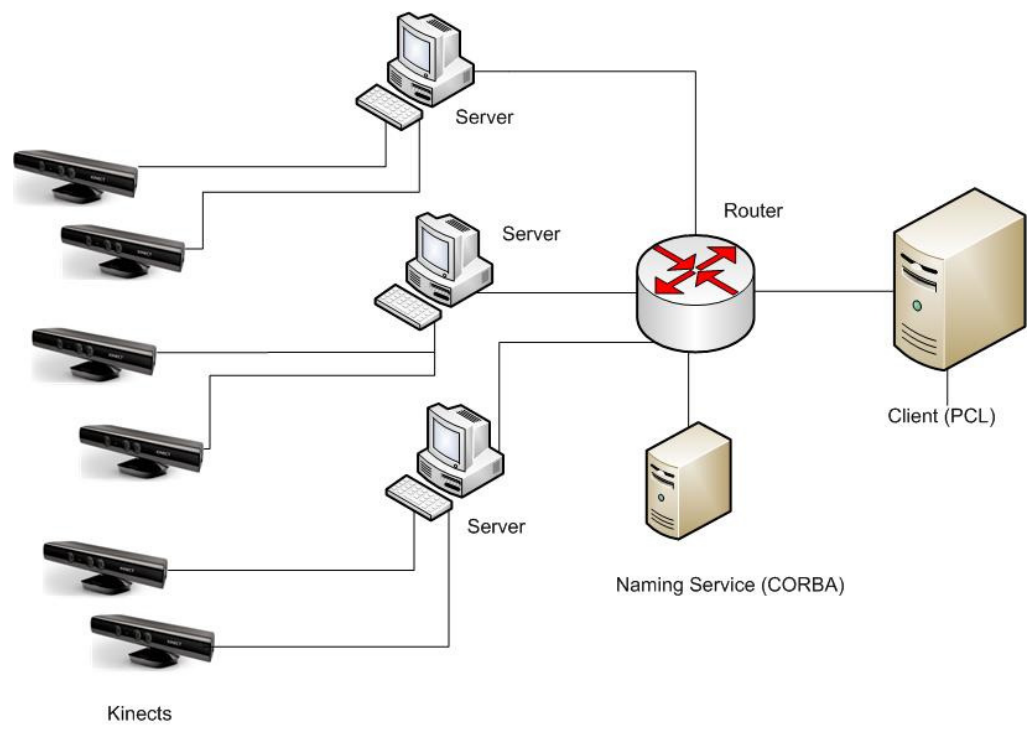

Figure 1.General scheme of the proposed system. 


\subsection{Data acquisition}

Kinect devices present certain limitations. First, its view range covers depth precisely only between $0.5 \mathrm{~m}$ and $3.5 \mathrm{~m}[9]$.

For this reason, one of the motivations of our system is to expand the covered view by adding several Kinects to the scenario. To add more Kinect devices to the scene, the naïve solution is to try to connect multiple cameras to the same computer. However, due to the large volume of data generated by each camera, a USB controller is needed to handle the bandwidth emitted by each one. As a consequence, that is not a valid solution, since most computers only support a limited number of USB controllers, usually two or three.The solution adopted for our system was to develop a distributed application with multiple cameras connected to multiple computers. In such a configuration, another important issue has to be taken into account. The infrared pattern emitted by different Kinects can interfere with each other, causing 'holes' in the acquired point clouds. Therefore, we must be careful in the placement of the devices and avoid placing a camera right in front of other one.

The data provided by each Kinect in which we are interested in are: a three-channel RGB imageof the scene, captured at a resolution of $640 \mathrm{x} 480 \mathrm{px}$; a depth map, which is a texture of the same resolution in which each pixel takes a value that indicates the distance between the infrared pattern and the sensor; a texture of user labels with same resolution, in which each pixel takes the value of the user id in front of the camera or a zero value; and the skeletons of the users in the scene, which are formed by joints representing the parts of the body (head, elbow, shoulders ...) andincludeboth xyz position and rotation from an initial pose position.

\subsection{Data coding}

Before the information captured by the remote devices can be sent, it has to be encoded. Kinect data to be processed includes RGB images, depth maps, user labels and skeleton joints.Skeleton joints information is sent without any compression, since the volume of data needed to store and transmit the position of all the joints is negligible compared to the volume of image or depth information.

To encode the RGB image we use a video compressor. It would be meaningless to use an image codec such as JPEG, since it only uses spatial information at the time of compression and thus the bit rate needed is much higher. For video compression, the proposed system uses the crossplatform library FFMPEG, which provides many audio and video codecs. The RGB image compression is done using the VP8 codec developed by Google TM [19] that needs a YUV 420 image format. Although VP8 codec introduces quality losses during compression and decompression, its balance between final quality and performance makes itadequate for our purposes. Additionally, the loss in quality remains quite low and the human eyes, acting as filters, are note able to appreciate it.

As it has been commented before, there is no compression codec to encode depth or a combination of RGB and depth information. In the proposed system, the compression of the depthmap has to be done in atricky way, based on the scheme proposed by Pece[20]. Basically, one depth channel has to be converted into a three-channel image, and then a specific codec $\mathrm{H} 264$ is used to compress the result. The H264 codec is more computationally expensive than VP8 and it also needs more bandwidth. However, the final results obtained for the particular case of depthinformation are much better than using VP8. 
Finally, for labels codification, the chosen codec was VP8. Using this codec, quality losses that could result in user misidentifications in the remote computer, can be expected. In order to prevent these situations, the following strategy is proposed. Since encoders usually join together colors being too close, we propose spacing them before codification. The values 0 to 15 of user labels are translated into values from 0 to 255, preventing the encoder to mix up them. In Figure 2 the conversion equivalencesare shown. With these new values, labels are storedinto a luminance channel and then compressed.

\begin{tabular}{cccccccccccccccc}
\hline $\mathbf{0}$ & $\mathbf{1}$ & $\mathbf{2}$ & $\mathbf{3}$ & $\mathbf{4}$ & $\mathbf{5}$ & $\mathbf{6}$ & $\mathbf{7}$ & $\mathbf{8}$ & $\mathbf{9}$ & $\mathbf{1 0}$ & $\mathbf{1 1}$ & $\mathbf{1 2}$ & $\mathbf{1 3}$ & $\mathbf{1 4}$ & $\mathbf{1 5}$ \\
\hline $\mathrm{I}$ & $\mid$ & $\mid$ & $\mid$ & $\mid$ & $\mid$ & $\mid$ & $\mid$ & $\mid$ & $\mid$ & $\mid$ & $\mid$ & $\mid$ & $\mid$ & $\mid$ & $\mid$ \\
$\mathbf{0}$ & 17 & 34 & 51 & 68 & 85 & 102 & 119 & 136 & 153 & 170 & 187 & 204 & 221 & 238 & 255 \\
\hline
\end{tabular}

Figure 2.Correspondence of user labels to colors to avoid misidentifications after data compresion.

Due to the computation requirements of the proposed system, in order to code and send the 3D information, it has been designed to process video sequences in parallel using threads. The RGB image, depth map, user labels and skeletons are acquired at the same time. Each type of data is then coded separately in parallel using the 'Boost' library.

\subsection{Data transmission}

System servers are registered in a CORBA naming service after starting, so that the system'scentralcomputer can find them, without needing to know their IP addresses. When the centralcomputer establish a communication and asks for data, the server collectsthe information from every local attached Kinect, encodes it and sends it continuously to the remote client. The client is constantly receiving data sent from each server, but it may not use all information that arrives to the client. The system is designed to decode only the information that is to be used. To this end, mutual exclusion techniques are employed.

Compressed information is stored into CORBA data arrays. Then, the server sends data by invoking remote methods in each client. These methods receive input arguments containingthe compressed RGB image, depth map, user labels and uncompressed skeletons. The information is sent only to the clients who have previously registered on the server.

\subsection{Point cloud fusion}

Once the Kinect cameras have been installed in the location to be monitored, a first system calibration has to be performed. The goal of calibration is for the central client computer to find the proper transformation matrices to align and fuse the received point clouds. One of devices is chosen to be the center of the coordinate system and then rotations are calculated from the other cameras. Given each pair of point clouds, the objective is to calculate a $4 \times 4$ rotation and translation matrices by solving the system of equations $\mathbf{B}=\mathbf{R A}+\mathbf{t}$, where $\mathbf{A}$ and $\mathbf{B}$ are threecomponentpoints, $\mathbf{R}$ is a $3 \times 3$ rotation matrix and $\mathbf{t}$ is a three-component columntranslation vector.

Within the system interface, the calibration step will prompt the user to check the correspondence of at least 3 common points in different clouds of points. This calibration clouds are not yet compressed for better results. For this purpose, it is useful to place an object into the intersection area of different infrared patterns. Figure 3 shows this process using points belonging to a chair and a box on top of it. Takingthe marked common points, the system can approximate an initial calibration, which serves to rotate the point clouds and apply the algorithm ICP (Iterative Closest Point), which refines the calibration. These rotation matrices have only to be computed the first 
time the system is deployed and they are later used to rotate all information coming from the different cameras, including user skeletons, in different executions.

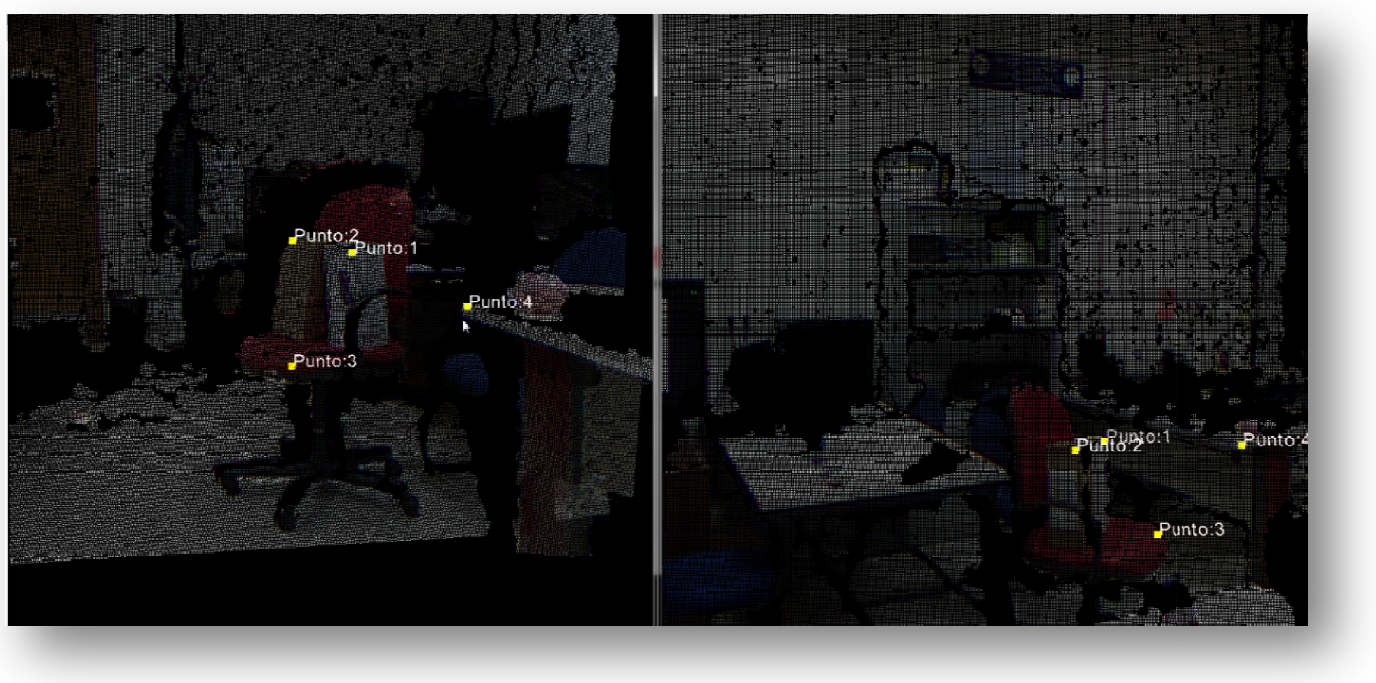

Figure 3.Initial calibration to determine rotation and translation matrices. These matrices are used to fuse 3D information coming from different Kinect cameras.

\subsection{Skeleton merging}

We distinguish between Kinect input skeletons and system's final output skeletons. Each output skeleton is computed dynamically from a linked list of input skeletons, which are merged and averaged together.

Figure 4 depicts the process of output skeletons computation. To merge the input skeletons, the first step is to apply rotation matrices to the detected joints. Once all the skeletons from all the cameras are in the same reference system, the algorithm for skeleton merging can be applied. The first step is to check for changes in the previous linked lists of skeletons, which contain the correspondence among similar input skeletons. These lists includethe camera identifiers, the input skeletons identifiers and the output skeletons identifiers.Every time a remote camera considers a Kinect skeleton to have disappeared, it is removed from its linked list. Accordingly, every time a camera provides information of a new skeleton, the system tries to add it into a linked list. To this end, it compares the distance between the joints representing the two skeleton heads. This strategy has been used in other human skeleton tracking proposals[21].If the distance is less than $15 \mathrm{~cm}$, the system considers both input skeletons to be the same output skeleton. Evaluating skeleton matching during 25 consecutive frames strengthens the robustness of the system. In case no correspondence can be found to include the new skeleton into any existing linked list, then it is considered as a new output skeleton and a new linked list is built up.The final joints are calculated by averaging all joints from different cameras. If the confidence of a specific joint within a skeleton is less than 0.5 , its position is not used to calculate final joint. The advantage of this design is that in case one camera cannot detect a given joint,its positioncan be determined from the information given of other camera. The probability of having all joints describing the skeleton available, and with accurate positions, grows with the number of cameras detecting the skeleton. 


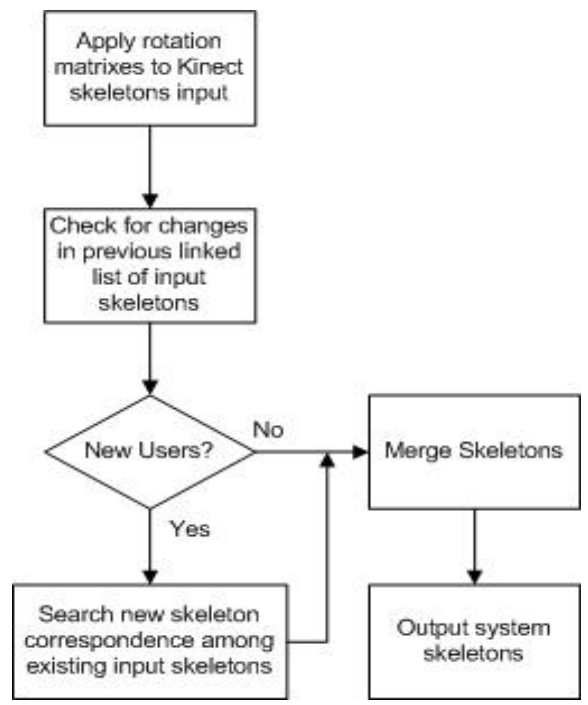

Figure 4.Skeleton merging algorithm.

\subsection{Data visualization and skeleton tracking}

The central client interface uses PCL to visualize the final reconstructed space and allows realtime tracking of labeled people inside the area covered by the cameras. Figure 5 shows a labeled skeleton being monitorized in real-time by 5 cameras. The final scene can be rotated and analyzed from any point of view. However, there is a limitation on the available frame rate due to the VTK rendering methods employed by the system. When the number of points in the final point cloud grows, the frame rate is reduced. This is not a problem related to the compression/decompression computational cost, but the visualization methods included in PCL. Future releases of PCL are said to address this problem by adding native OpenGL rendering [17]. In order to guarantee usability of the system, despite of this problem, the user interface allows subsampling the number of points to visualize. Test and results section gives some figures of performance with 5 device cameras.

Finally, the system is designed to store skeleton information of peoplewithin the tracking area, associated to their labeled output skeletons. Once recording has started, all the user jointsare stored in a raw file that can be further used to reproduce any situation occurred or serve as an input for another application for further situation analysis (i.e. movement recognition application). For applications that require human activity registration, the needed storage space is much smaller than in conventional $2 \mathrm{D}$ video systems, since only the skeletons may need to be stored.

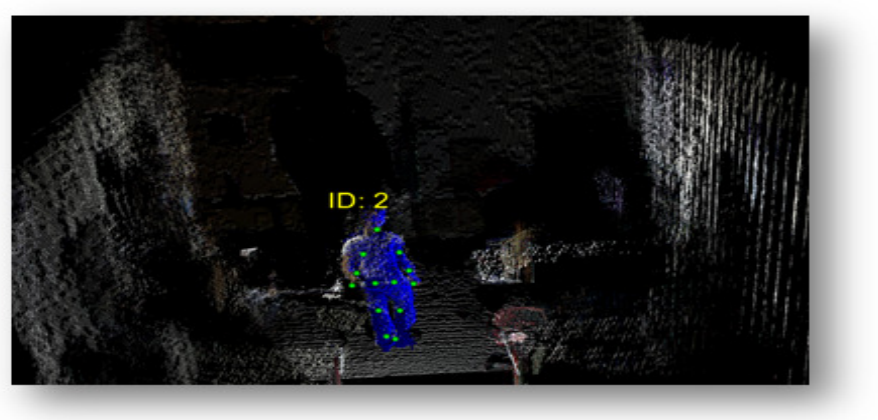

Figure 5.Labeled skeleton and associated joints obtained from the combination of 5 cameras information. 


\section{TESTS AND RESULTS}

The system has been tested using 5 Kinect cameras connected to three personal computers: two desktop computers equipped with an Intel i5-2400 CPU running at $3.10 \mathrm{GHz}$ and a laptop equipped with an Intel $17-3610 \mathrm{QM}$ CPU running at $2.30 \mathrm{GHz}$. The client computer was an Intel Xeon X5650 with 24 cores running at 2.66 GHzand equipped with aNvidia GeForce GTX580 GPU. The server computers were running Windows 7 operating system, while the central client was running Linux Fedora 16.

The aim of these tests was to check the performance of the final system in real conditions. The first tests conducted included data transmission, coding/decoding and visualization measurements.Using RGB input at $640 \times 480 \mathrm{px}$ resolution and coding depth information to $320 \times 240$ px color maps, the theoretical limit on the number of cameras that can be connected over a Gigabit Ethernet is higher than 50 for a framerate of $30 \mathrm{fps}$. These numbers do not consider the overhead of TCP connection. In our experimental tests, performed with up to 5 cameras (the maximum number of cameras we managed to have), the obtained framerate was actually $30 \mathrm{fps}$. However, in our tests, we detected that even having every server transmitting at $30 \mathrm{fps}$ and the client computer decoding all cameras information at the same framerate, the final scene rendering was affected by VTK visualization limitations of PCL. As explained above, the achieved frameratedependson the number of points in the cloud. Table 1 shows how visualizing a cloud of points constructed from 5 cameras renders only at $7 \mathrm{fps}$ if every point is drawn onto the screen. Subsampling the number of points by 16 , which actually still provides a very nice representation of the scene, improves performance to $29 \mathrm{fps}$.

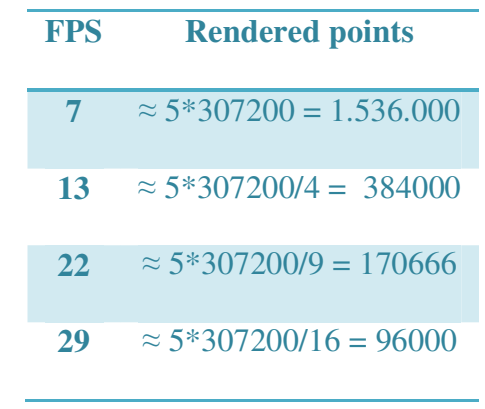

Table1. Frame rate obtained during visualization using VTK for 5 cameras 3D reconstruction. This is a limitation of VTK, not the system itself.

The second battery of tests conducted included situations to measure the behavior of the system with different people in the scene and measure the robustness to self-user and inter-user occlusions. The first test consisted in a user placed in the center of the scene. Meanwhile, another user revolves around him or her, so that some cameras can see the first user and some others cannot.The goal is to test the robustness of the system when different cameras detect and lost Kinect skeletons over and over again. The test was conducted ten times using combinations of different height users and the obtained result was always successful in every situation, since the system did not confuse users or incorrectly merged their skeletons. The second test consisted in users sitting and getting up from chairs in an office space. This test measured the robustness of the system to some skeletons joint occlusions, since some of the cameras are not able to provide accurate positions for body parts behind tables or chairs. The test was repeated for ten different people sitting in front of the four tables in the scene in Figure 6 and again the system worked perfectly. The third test consisted in covering and uncovering one by one the different cameras in the scene while 5 people were being tracked in the scene. The goal was to test what happens when 
multiple Kinect input skeletons are removed and detected at the same time. The result was again satisfactory and every computed output skeletons in the scene kept being tracked consistently.

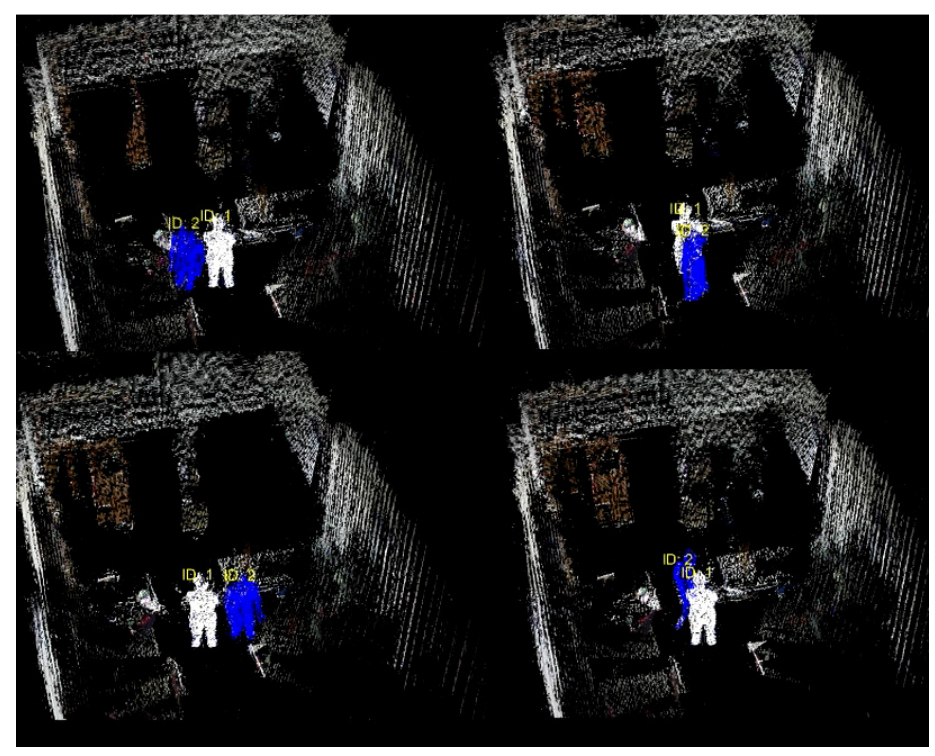

Figure 6 Inter-user occlusion test within a space monitorized by 5 cameras.

\section{CONCLUSIONS}

This article describes a distributed CORBA system for remote space 3D monitoring using Kinect consumer depth cameras. Due to the high bandwidth needs of these cameras, the maximum number of cameras that can be connected to a single computer is usually two. The solution provided in this paper includes a client-server application that can handle the information acquired by any number of cameras connected to any number of computer servers. Since one Kinect camera can only detect precisely the depth information within a field of view of 3.5 meters, the proposed system solves, at the same time, the limitation on the size of the location that can be monitorized precisely. A central client computer can be used to monitor the reconstructed 3D space in real time and track the movements of people within it.

In the central client computer, a skeleton-merging algorithm is used to combine the information of skeletons belonging to the same person, but generated by different Kinect cameras, into a single output skeleton. The tests conducted showed that this algorithm is robust under several situations, avoiding unwanted duplication of skeletons when new people enter the scene or under camera or inter-user occlusions. Moreover, the algorithm combines the information coming from each skeleton joint independently, so the 3D location of joints in the final generated skeleton is more precise, having been averaged among all the cameras detecting that joint. In case a self-user or a inter-user occlusion causes one joint not to be detected by one or more of the cameras,its position is reconstructed using the information coming from cameras in which the joint has been detected with enough confidence. Output skeleton movements can be stored in raw files for further analysis of situations.

Thissystem provides a very precise and convenient way of monitoring a 3D space at an affordable price. People activity in the scene can be registered for further analysis and the storage needs to keep track of human behavior under different circumstances can be much lower than for 
conventional 2D systems, if only the skeletons are needed. Future research tasks will include designing a top activity recognition layer that could monitor people behavior and interactions.

\section{ACKNOWLEDGEMENTS}

This research was supported in part by the Ministerio de Ciencia e Innovación under project TIN2010-20529 and Junta de Castilla y León under project VA171A11-2.

\section{REFERENCES}

[1] Sage, K. \& Young S. (1999) Security Applications of Computer Vision, Aerospace and Electronic Systems Magazine, IEEE 14(4):19-29.

[2] Boutaina, H., Rachid, O.H.T. \&Mohammed, E.R.T. (2013) Tracking multiple people in real time based on their trajectory, in Proc. of Intelligent Systems: Theories and Applications (SITA), 8th International Conference on, pp.1-5, Rabat, Morocco.

[3] Strbac, M., Markoviu, M., Rigolin, L. \& Popoviu, D.B. (2012) Kinect in Neurorehabilitation: Computer vision system for real time and object detection and instance estimation, in Proc. Neural Network Applications in Electrical Engineering (NEUREL), 11th Symposium on, pp. 127-132, Belgrade, Serbia.

[4] Martín Moreno, J., Ruiz Fernandez, D., Soriano Paya, A. \& Berenguer Miralles, V. (2008) Monitoring 3D movements for the rehabilitation of joints in Physiotherapy, in Proc. Engineering in Medicine and Biology Society (EMBS), 30th Annual International Conference of the IEEE, pp. 48364839, Vancouver, Canada.

[5] Ukita, N. \& Matsuyama, T. (2002) Real-Time Cooperative Multi-Target Tracking by Communicating Active Vision Agents, in Proc. Pattern Recognition, 16th International Conference on, vol.2, pp.1419.

[6] RBodor, R., Jackson, B.\&Papanikolopoulos, N. (2003) Vision-Based Human Tracking and Activity Recognition, in Proc. of the 11th Mediterranean Conf. on Control and Automation, pp. 18-20.

[7] Poppe R. (2007). Vision-based human motion analysis: An overview,Computer Vision and Image Understanding 108:4-18.

[8] Weinland, D., Ronfard, R.\& Boyer, E. (2011) A survey of vision-based methods for action representation, segmentation and recognition, Computer Vision and Image Understanding115(2):224241.

[9] Kinect for Windows (2013)Kinect for Windows. Retrieved from http://www.microsoft.com/enus/kinectforwindows/develop/overview.aspx, last visited on July 2013.

[10] Burrus, N. (2013) RGBDemo. Retrieved from http://labs.manctl.com/rgbdemo/, last visited on July 2013.

[11] KinectTCP (2013)KinectTCP. Retrieved from https://sites.google.com/a/temple.edu/kinecttcp/, last visited on July 2013

[12] OpenNI (2013) OpenNI. Plataform to promote interoperability among devices, applications and Natural Interaction (NI) middleware. Retrieved from http://www.openni.org, last visited on July 2013.

[13] Prime Sense (2013) Prime Sense. Retrieved from http://www.primesense.com/, last visited June 2013.

[14] Martínez Rach, M.O., Piñol, P., López Granado, O. \& Malumbres, M.P. (2012) Fast zerotree wavelet depth map encoder for very low bitrate, in Actas de las XXIII Jornadas de Paralelismo, Elche, Spain.

[15] Joon-Heup, K., Moon-Sang J. \& Jong-Tae, P. (2001) An Efficient Naming Service for CORBA-based Network Management, in Integrated Network Management Proceedings, IEEE/IFIP International Symposium on, pp.765-778, Seattle, USA

[16] PCL (2013) Point Cloud Library. Retrieved from http://pointclouds.org/, last visited on July 2013.

[17] PCL Developers Blog (2013) PCL Developers Blog. Retrieved from http://pointclouds.org/blog/, last visited on July 2013.

[18] Parajuli, M., Tran, D.; Wanli, Ma; Sharma, D. (2012) Senior health monitoring using Kinect, in Proc. Communications and Electronics (ICCE), Fourth International Conference on, pp. 309-312, Hue, Vietnam.

[19] The WebM Project (2013) The WebM Project. Retrieved from http://www.webmproject.org, last visited on July 2013. 
[20] Pece, F., Kautz, J. \& Weyrich, T. (2011) Adapting Standard Video Codecs for Depth Streaming, in Proc. of the 17th Eurographics conference on Virtual Environments \& Third Joint Virtual Reality (EGVE - JVRC), Sabine Coquillart, Anthony Steed, and Greg Welch (Eds.), pp. 59-66, Aire-la-Ville, Switzerland.

[21] García, J., Gardel, A., Bravo I., Lázaro, J.L., Martínez, M. \& Rodríguez, D. (2013). Directional People Counter Based on Head Tracking, IEEE Transactions on Industrial Electronics 60(9): 39914000.

\section{Authors}

Mario Martínez-Zarzuela was born in Valladolid, Spain; in 1979. He received the M.S. and $\mathrm{Ph} . \mathrm{D}$. degrees in telecommunication engineering from the University of Valladolid, Spain, in 2004 and 2009, respectively. Since 2005 he has been an assistant professor in the School of Telecommunication Engineering and ar e searcher in the Imaging\& Telematics Group of the Department of Signal Theory, Communications and Telematics Engineering. His research interests include parallel processing on GPUs, computer vision, artificial intelligence, augmented and virtual reality and natural human-computer interfaces.

Miguel PedrazaHueso was born in Salamanca, Spain, in 1990, He received his title in Tele communication Engineering from the University of Valladolid, Spain, in 2013.

Since 2011, he has collaborated with Imaging \& Telematics Group of the Department of Signal Theory, Communications and Telematics Engineering. His research interests include applications with Kinect and augmented reality.

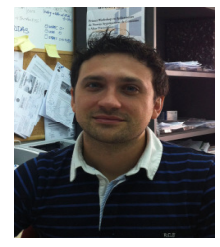

Francisco Javier Diaz-Pernas was born in Burgos, Spain, in1962.Hereceivedthe Ph.D. de gree in industrial engineering from Valladolid University, Valladolid, Spain, in 1993. From 1988 to 1995, he joined the Department of System Engineering and Automatics, Valladolid University, Spain, where he has worked in artificial vision systems for industry applications as quality control for manufacturing. Since 1996, he has been a professor in the School of Telecommunication Engineering and a Senior Researcher in Imaging \& Telematics Group of the Department of Signal Theory, Communications, and Telematics Engineering. His main research interests are applications on the Web, in telligent transportation system, and neural networks for artificial vision.

David González-Ortega was born in Burgos, Spain, in 1972. He received his M.S. and $\mathrm{Ph} . \mathrm{D}$. degrees in telecommunication engineering from the University of Valladolid, Spain, in 2002 and 2009, respectively. Since 2003 he has been a researcher in the Imaging and Telematics Group of the Department of Signal Theory, Communications and Telematics Engineerin g. Since 2005, he has been an assistant professor in the School of Telecommunication Engineering, University of Valladolid. His research interests include computer vision, image analysis, pattern recognition, neural networks and real-time applications.

MíriamAnton-Rodriguez was born in Zamora, Spain, in 1976. She received her M.S. and $\mathrm{Ph} . \mathrm{D}$. degrees in telecommunication engineering from the University of Valladolid, Spain, in 2003 and 2008, respectively. Since 2004, she is an assistant professor in the School of Telecommunication Engineering and a researcher in the Imaging \& Telematics Group of the Department of Signal Theory, Communications and Telematics Engineering. Her teaching and research interests includes applications on the Web and mobile apps, bio-inspired algorithms for data mining, and neural networks for artificial vision.
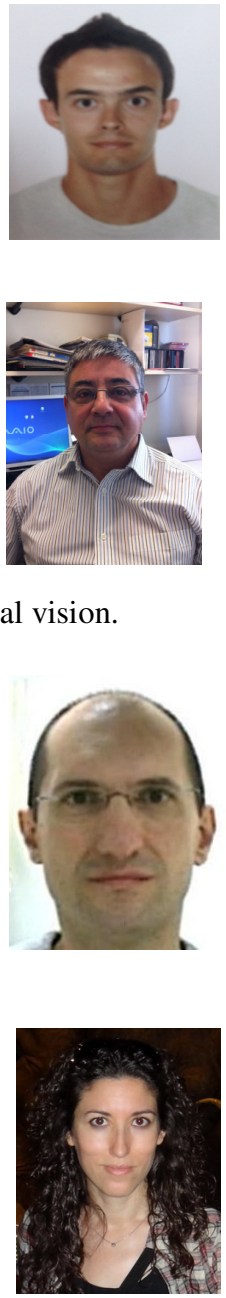